\title{
General Equivalency Diploma Completion
}

National Cancer Institute

\section{Source}

National Cancer Institute. General Equivalency Diploma Completion. NCI Thesaurus.

Code 667135.

Indicates that a person has received a General Equivalency Diploma in lieu of a high school diploma. 